\title{
Full Mouth Rehabilitation of a Patient with Severely Worn Dentition and Reduced Occlusal Vertical Dimension: A Clinical Report
}

Bipin Yeshwantrao Muley, Arun N Khalikar, Sameera Rafique Shaikh, Mohana M Tagore, Pravinkumar G Patil, Sattyam V Wankhade

\begin{abstract}
Esthetic and functional rehabilitation of severely worn dentition presents a significant challenge to the restorative dentist. The prerequisites in restoring the worn dentition are to understand the determinants of the occlusal vertical dimension and the effects of its alteration on the temporomandibular joint, muscle comfort, bite force, speech and long-term occlusal stability. This clinical report describes a sequential treatment procedure of full mouth rehabilitation of a patient with severe attrition and cervical abrasion of teeth with metal-ceramic restorations and implant supported fixed dental prosthesis after establishing a functional and esthetic vertical dimension and occlusal plane.
\end{abstract}

Keywords: Attrition, Full mouth rehabilitation, Occlusal vertical dimension, Wear.

How to cite this article: Muley BY, Khalikar AN, Shaikh SR, Tagore MM, Patil PG, Wankhade SV. Full Mouth Rehabilitation of a Patient with S everely W orn Dentition and Reduced Occlusal Vertical Dimension: A Clinical Report. Int J Prosthodont Restor Dent 2012;2(2):72-76.

Source of support $\mathrm{Nil}$

Conflict of interest: None

\section{INTRODUCTION}

Tooth wear is an increasingly important clinical problem in aging populations, especially with the increased life expectancy. ${ }^{1,2}$ A ccording to Dawson, the basic mechanism for tooth surface deformation results from three basic physical and chemical mechanisms namely: (i) Stress, (ii) friction which includes abrasion and attrition, and (iii) corrosion. ${ }^{3}$ Tooth wear can result from abrasion, attrition and erosion. ${ }^{4-8}$ Tooth wear observed in any individual may be the result of a combination of all the possible etiological factors over lifetime of the dentition. ${ }^{9}$ The management of tooth wear, especially attrition, is becoming crucial, both from a preventive and a restorative point of view. ${ }^{10,11}$ Comprehensive treatment planning along with assessment of vertical dimension at rest and in occlusion is required for each individual clinical condition. A rticulated study casts and diagnostic wax-up provide necessary information for the evaluation of treatment options. Diagnostic splints or provisional restorations itself are used to assess the tolerance of the patient to changes in occlusal vertical dimension (OVD). ${ }^{12}$ Continuous tooth eruption and alveolar growth usually compensates the loss of OV D caused by physiologic tooth wear. ${ }^{13} \mathrm{~W}$ hen the tooth wear exceeds the compensatory mechanism, there is loss of OVD. However, more commonly, the rate of tooth wear is slow and compensatory eruption of the opposing teeth eliminates space for restoration. ${ }^{14}$ Restoration of such worn teeth becomes a challenge as the space availability for prostheses becomes limited. The prerequisites in restoring worn dentition are to understand the determinants of the OVD and the effects of its al teration on the temporomandibular joint (TM J), muscle comfort, bite force, speech and long-term occlusal stability. ${ }^{15}$ Best vertical dimension is one that satisfies the patient's esthetic desires and the clinician's functional goals with most conservative approach. ${ }^{16}$ The objective of complete mouth rehabilitation is reconstruction, restoration and maintenance of health of the entire stomatognathic system. Complete mouth rehabilitation is a dynamic, functional problem and embodies the correlation and integration of all the component parts into one functioning unit. The aim and endeavor, therefore, must be reconstruction and rehabilitation of the whole, satisfying all the related factors. ${ }^{17}$

The present article describes a systematic approach in the treatment of a patient with severely worn dentition using full mouth metal-ceramic restorations and implant supported fixed dental prosthesis (FDP) after establishment of a functionally stable, esthetic occlusal plane using B roderick occlusal plane analyzer.

\section{CLINICAL REPORT}

A 40-year-old male patient came to the Department of Prosthetic Dentistry with a chief complaint of anterior teeth wear and requested esthetic enhancement (Fig. 1A ). Clinical and radiographic examinations revealed generalized severe attrition and cervical abrasion (Figs $1 B$ and C). M ore aggressive attrition was seen in mandibular dentition and the maxillary teeth showed supreruption; therefore the occlusal plane appeared to be irregular and at a lower level. $M$ andibular central incisors and left lateral incisor were missing. TMJ function was normal with no clicking or tenderness. The patient denied of having any parafunctional habit but the relatives pointed out bruxism and night grinding. The patient gave history of brushing teeth with 
Full Mouth R ehabilitation of a Patient with Severely Worn Dentition and R educed Occlusal Vertical Dimension

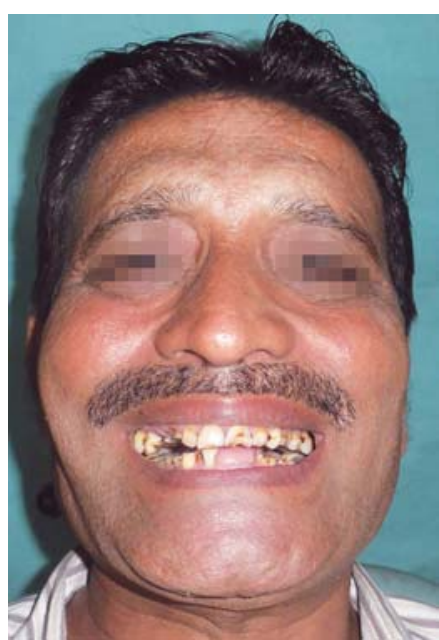

Fig. 1A: Pretreatment profile view

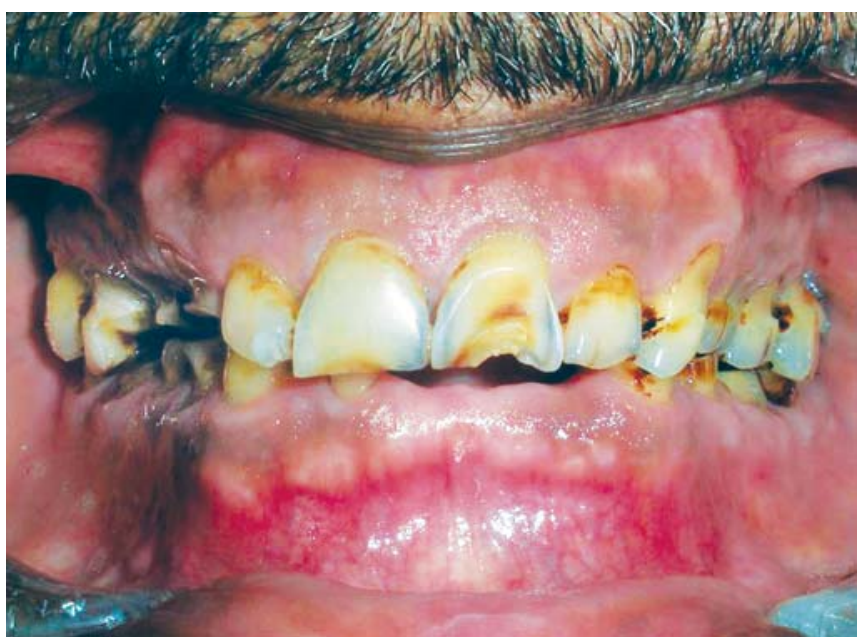

Fig. 1B: Pretreatment frontal view of teeth in occlusion

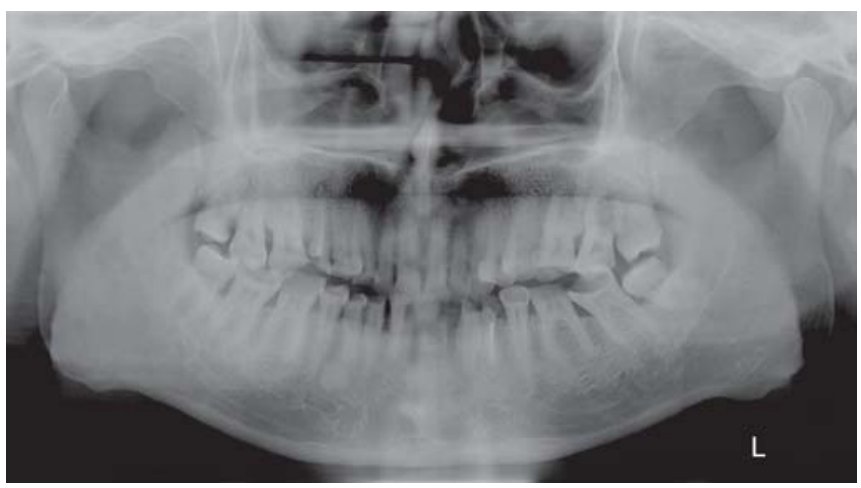

Fig. 1C: Pretreatment radiograph

an abrasive tooth powder with a hard bristle brush. After complete diagnostic evaluation, treatment plan was designed to establish the occlusal plane at a proper level and full mouth rehabilitation of the maxillary and mandibular natural dentition with metal-ceramic restorations after endodontic treatment and implant supported FDP in place of the missing mandibular incisors. The patient was instructed about proper brushing technique with a less abrasive toothpaste and soft bristle brush.

Study casts were obtained and mounted on a semiadjustable articulator (B io-A rt, Sao Carlos, Sao Paulo, $B$ razil) using centric relation occlusal record and a facebow transfer. As revealed from diagnostic mounting, there was insufficient height of several teeth. Endodontic therapy of all natural teeth and crown lengthening procedure of several maxillary and mandibular teeth was carried out for longterm functional and esthetic restorations of the teeth. Maxillary left second and third molars showed poor prognosis and were advised for extraction. A centric relation anterior index from pattern resin (GC Corporation, Tokyo, Japan) was fabricated to maintain the centric relation position at the desired OV D during teeth preparation phase. M ock preparation and diagnostic wax-up of anterior teeth was accomplished. By using Broderick's occlusal plane analyzer, occlusal plane was determined on the mandibular cast (Figs 2A and B) and a baseplate wax (modeling wax; Deepti Dental Products, Ratnagiri, India) occlusal plane index was made on this established occlusal plane. ${ }^{18}$ The occlusal plane index was mounted in place of maxillary cast and used to prepare diagnostic wax-up of mandibular posterior teeth at the established occlusal plane (Fig. 2C). Maxillary posterior wax-up was completed using the mandibular wax-up (Fig. 2D). Vinyl polysiloxane putty (Elite HD +, Zhermack SpA , B adia Polesine, Italy) templates were made of the diagnostic wax-up. Preparation of the maxillary and mandibular teeth was performed using the centric relation index to confirm clearance. Postcore restorations with glass fiber posts (DT Light-Post Illusion, Bisco Inc, Illinois, USA ) was cemented with maxillary right canine, first and second premolars (Fig. 3). Core build of maxillary and mandibular teeth was accomplished with composite resin ( $M$ atrix, M edicept D ental, M iddlesex, UK). By using putty templates of the diagnostic wax-up, provisional restorations were fabricated from bis-acryl resinbased provisional restorative material (Luxatemp Star,

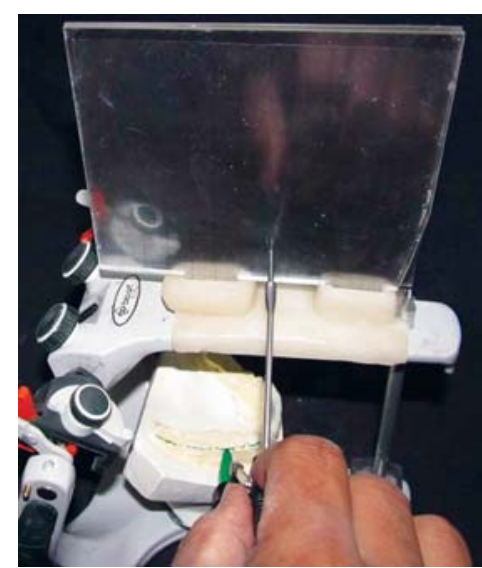

Fig. 2A: O cclusal plane determined using Broderick occlusal plane analyzer

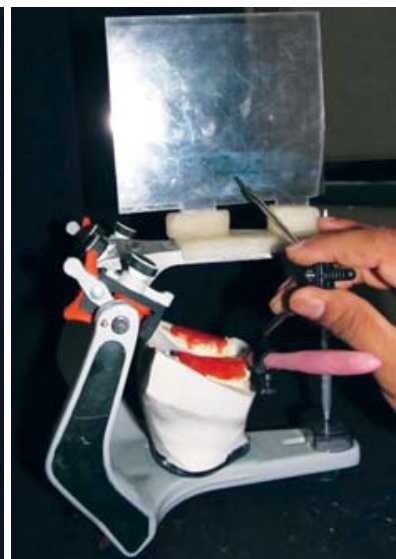

Fig. 2B: Occlusal plane carved on baseplate wax block 


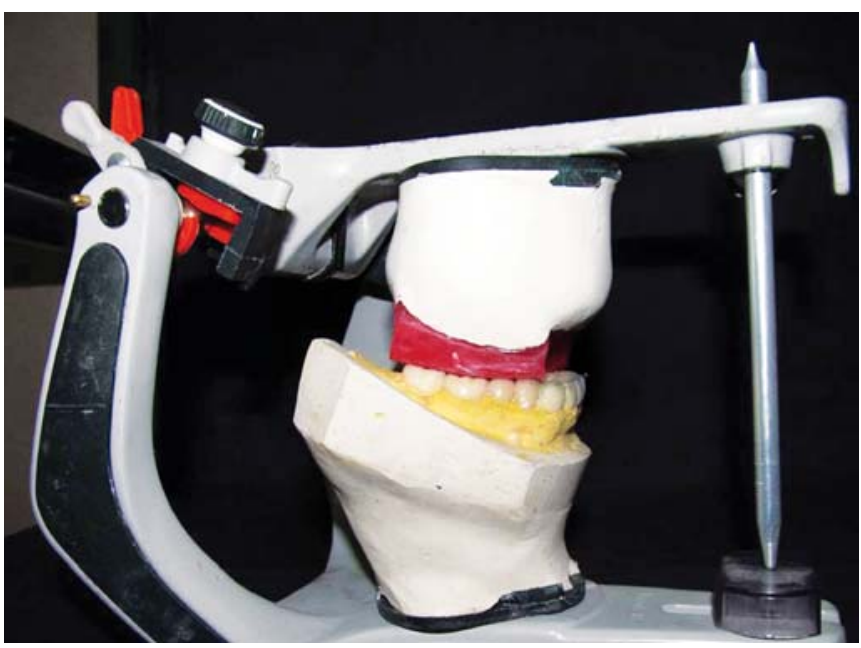

Fig. 2C: Occlusal plane index mounted and mandibular diagnostic wax-up accomplished

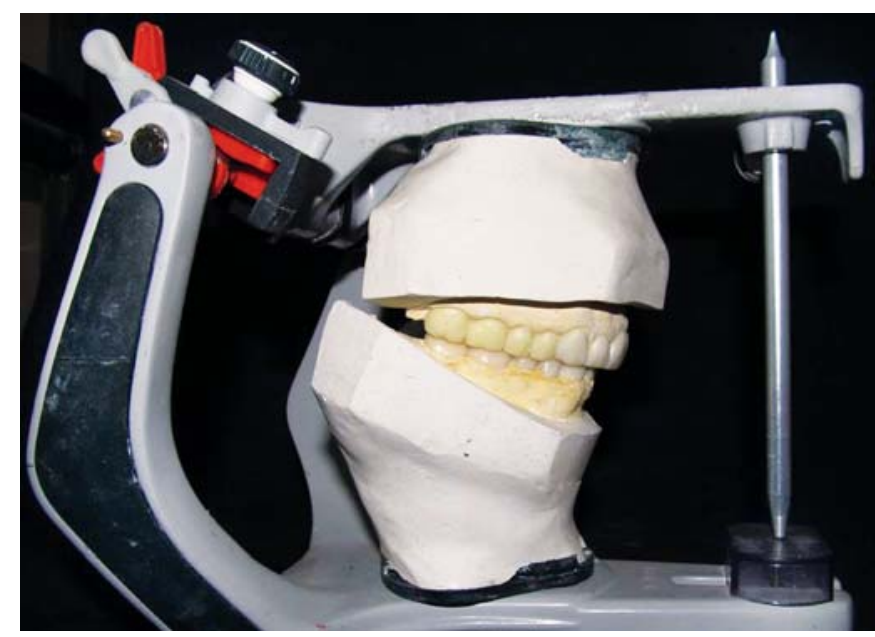

Fig. 2D: Maxillary posterior wax-up

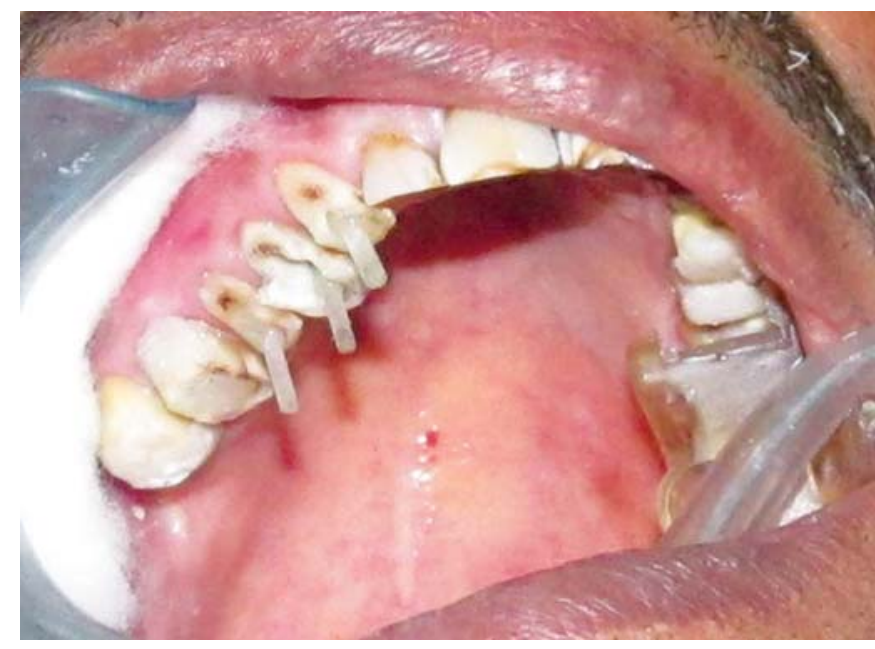

Fig. 3: Post-core with glass fiber posts in maxillary right canine, first and second premolars

DM G , H amburg, G ermany) and cemented using temporary cement (TempoCem Ne, DM G, Hamburg, Germany). The missing mandibular incisors were replaced with fixed provisional dental prosthesis at this time. The provisional restorations were subsequently equilibrated to establish maximum intercuspation in centric relation along with canine guidance and anterior coupling in protrusive guidance.

Once the provisional restorations were equilibrated and esthetics and phonetics were deemed satisfactory, an occlusal bite record was made of the maxillary and mandibular provisional restorations. Impressions of the provisional restorations made, casts were mounted using facebow and occlusal bite record of the provisional restorations against each other. A custom incisal guide table was fabricated to reproduce the anterior guidance established with the provisional restorations. The protrusive path and lateral excursions were recorded in putty on the flat guide table by movement of the articulator pin. A vinyl polysiloxane putty (Elite HD+, Zhermack SpA, Badia Polesine, Italy) index was made from the provisional models to duplicate the provisional restorations in definitive restorations. Patient used these provisional restorations for 3 months and was evaluated weekly for occlusion, wear and TMJ tolerance. After 3 months, the provisional restorations were removed and teeth were modified to make the definitive impressions with vinyl polysiloxane impression material (Elite HD+, Zhermack SpA, Badia Polesine, Italy) in a custom acrylic tray. $V$ inyl polysiloxane bite registration paste (Exabite II NDS, GC A merica INC, Alsip, Illinois, USA) was injected between anterior teeth while keeping the posterior provisionals in place. Two posterior bite registration records were obtained by placing the anterior bite record and keeping provisional restorations on one side. In this way three individual bite registration records (one of anterior sextant and two of posterior sextants) were obtained while maintaining the OVD achieved by provisional restorations. After recording the facebow relation, definitive casts were mounted on semiadjustable articulator. The wax-up for the definitive restorations were prepared using cut back technique using custom incisal guide table and putty index of provisional casts, and definitive metal-ceramic restorations were fabricated. Unglazed metal-ceramic definitive restorations were tried in the patient's mouth and evaluated for esthetics, occlusion and phonetics. After complete satisfaction of the patient, the restorations were glazed and cemented using glass ionomer luting cement (GC Gold Label, GC Corporation, Tokyo, J apan). Osteotomy was performed in the region of mandibular right central incisor and left lateral incisor to receive two one-stage implants (Fig. 4). I mplant supported provisional FDP was prepared and cemented on the implants. The provisional FDP was replaced with a definitive metal-ceramic FDP after 3 months of evaluation (Fig. 5A). Posterior disclusion on protrusion and canineguided occlusion on lateral excursion was achieved in the 


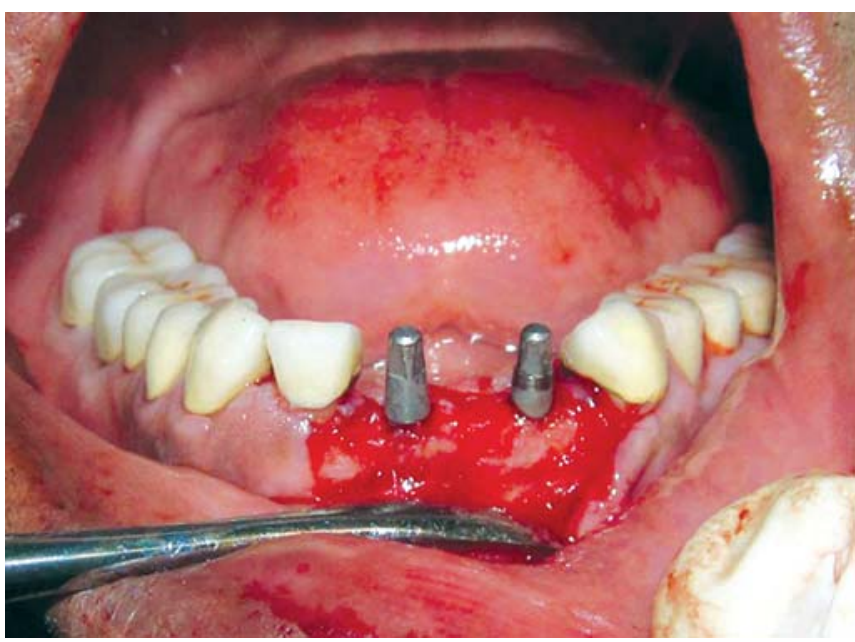

Fig. 4: Two one-stage implants in place of mandibular right central incisor and left lateral incisor

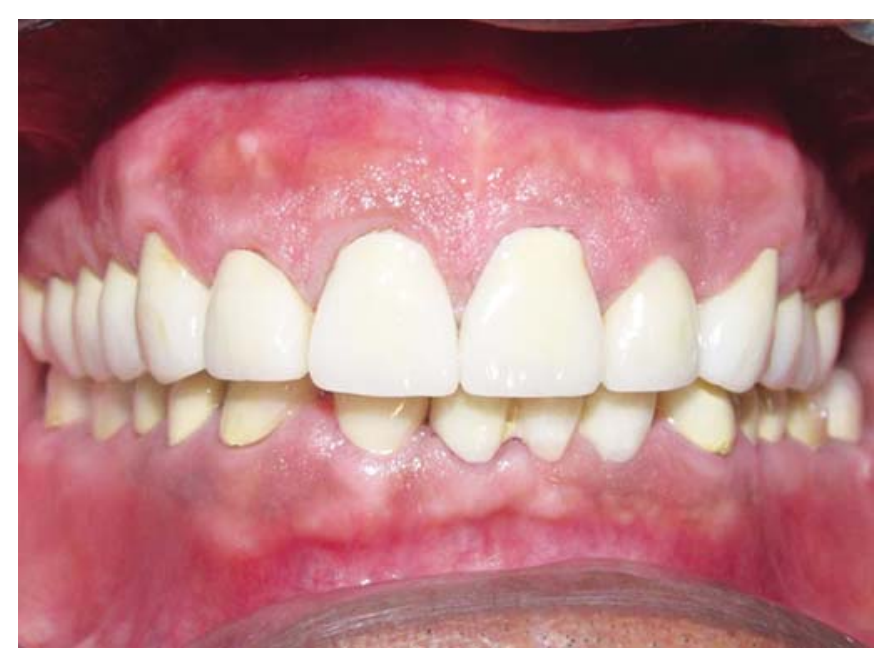

Fig. 5A: Definitive restorations in left lateral excursion

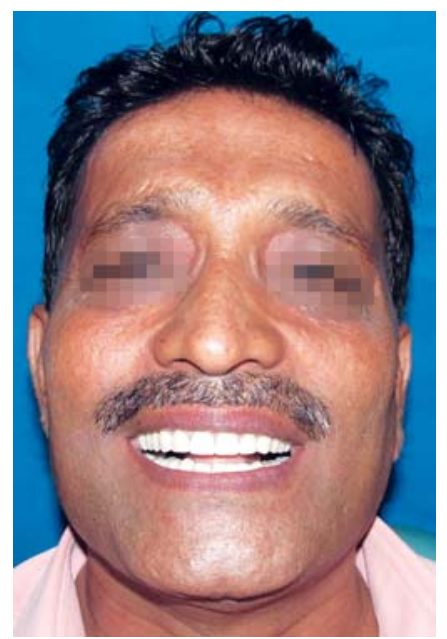

Fig. 5B: Post-treatment profile view

definitive restorations. The patient was given oral and written oral hygiene instructions and recalled for periodic examination. Patient was satisfied with the esthetic and functional outcome of the treatment after 1 year follow-up (Figs 5B and C).

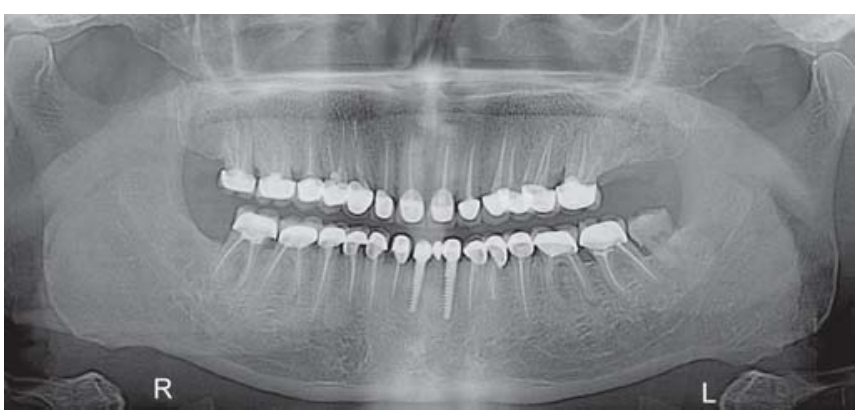

Fig. 5C: Post-treatment radiograph

\section{DISCUSSION}

Tooth surface loss has been classified into erosion, attrition, abrasion and abfraction. ${ }^{4}$ Tooth wear has a multifactorial cause and may be generalized throughout the dentition, but is often localized to incisors and canines..$^{19}$ The distribution of wear in the dentition is not even, as is evidenced by the difference between anterior and posterior teeth. ${ }^{20}$ I nadequate or unstable posterior support has been identified as a factor in severe anterior attrition and decreased OVD. ${ }^{21}$ Posterior occlusal prematurities, too, may cause increased function on anterior teeth, resulting in increased wear. Clinical judgment plays a major role in the assessment of this important component in rehabilitation. A variety of techniques, such as phonetics, interocclusal distance, swallowing and patient preferences, have been proposed to determine measurements for the correct OV D. ${ }^{22,23}$

It is important to establish the cause of wear before intervention to help improve the effectiveness of any preventive and restorative care. ${ }^{19}$ The patient in the clinical report had severe attrition and cervical abrasion of teeth. Although, the patient denied of having bruxism or night grinding habit, but his relatives pointed out these parafunctional habits. B ruxism control through the use of a nightguard by rigid occlusal stabilization appliance relieved in the region of implants was provided to reduce harmful stresses on implants and rest of the dentition. ${ }^{24}$ Patient was also having cervical abrasion so he was advised of proper brushing technique using soft bristle toothbrush and a toothpaste with less amount of abrasive agents. Full mouth endodontic therapy was essential part of the treatment procedure as the dentition was having severe attrition and dentin exposure. Endodontic treatment also helped in preparing the teeth to receive restorations with sufficient retention and to reduce post-treatment sensitivity or other complications.

\section{SUMMARY}

Severe wear cases present many challenges to the restorative dentist, including gaining the space to create restorations 
to satisfy the patient's esthetic desires, while also fulfilling occlusal and functional parameters that are essential for long-term success. The maintenance of severe wear cases can be ensured by the development of proper anterior guidance that allows for posterior disclusion within the patient's envelope of function. Taking this guidance into account during provisionalization ensures minimal adjustments in the definitive restorations and a greater longterm predictability of the case. This article elucidated a patient with severe attrition, abrasion and missing teeth. Full mouth rehabilitation of the patient with metal-ceramic restorations and implant supported FDP was described in detail.

\section{REFERENCES}

1. Smith $B G, K$ night JK. A $n$ index for measuring the w ear of teeth. BrDent J 1984;156:435-38.

2. Bartlett D, Phillips K, Smith B . A difference in perspective-the North A merican and European interpretations of tooth wear. Int J Prosthodont 1999;12:401-08.

3. Dawson PE. Functional occlusion from TMJ to smile design. Missouri: Mosby Inc 2007;17-26.

4. A ddy M , Shellis RP. Interaction between attrition, abrasion and erosion in tooth wear. M onogr Oral Sci 2006;20:17-31.

5. B eyth N, Sharon E, Li ipovetsky M, Smidt A. W ear and different restorative materials: A review. Refuat Hapeh $V$ ehasshinayim 2006;24:6-14.

6. Grippo J 0, Simring M, Schreiner S. Attrition, abrasion, corrosion and abfraction revisited: A new perspective on tooth surface lesions. J A m Dent A ssoc 2004;135(8):1109-18.

7. Verrett RG. A nalyzing the etiology of an extremely worn dentition. J Prosthodont 2001;10(4):224-33.

8. Litonjua LA, A ndreana S, Bush PJ, Cohen RE. Tooth wear: Attrition, erosion, and abrasion. Q uint Int 2003;34(6):435-46.

9. Crothers AJR. Tooth wear and facial morphology. J Dent 1992;20:333-41.

10. Spijker AV, K reulen CM , Creugers NHJ . A ttrition, occlusion, (dys)function, intervention: A systematic review. Clin Oral Impl Res 2007;18:117-26.

11. Hemmings K W, Howlett J A, W oodley NJ, et al. Partial dentures for patients with advanced tooth wear. Dent U pdate 1995;2:52-59.

12. Ibbetson RJ, Setchell DJ. Treatment of the worn dentition: 2 . Dent U pdate 1989;16:305-07.

13. M urphy T. Compensatory mechanisms in facial height adjustment to functional tooth attrition. A ust Dent J 1959;4:312-23.

14. Berry DC, Poole DF. Attrition: Possible mechanisms of compensation. J Oral Rehabil 1976;3:201-06.

15. Lerner J. A systematic approach to full-mouth reconstruction of the severely worn dentition. Pract Proced A esthet Dent 2008;20(2):81-87.
16. Spear FM. A pproaches to vertical dimension. Adv Esthet Interdiscip D ent 2006;2(3):2-12.

17. K azis $\mathrm{H}$. Functional aspects of complete mouth rehabilitation. J Prosthet Dent 1954:4:833-41.

18. Bedia SV, Dange SP, K halikar AN. Determination of the occlusal plane using a custom-made occlusal plane analyzer: A clinical report. J Prosthet Dent 2007;98:348-52.

19. B artlett DW, Shah P. A critical review of non-carious cervical (wear) lesions and the role of abfraction, erosion and abrasion. J Dent Res 2006;85:306-12.

20. M oslehifard E, Nikzad S, Geraminpanah F, M ahboub F. Fullmouth rehabilitation of a patient with severely worn dentition and uneven occlusal plane: A clinical report. J Prosthodont 2012;21:56-64

21. Turner KA, M issirlian DM. Restoration of the extremely worn dentition. J Prosthet Dent 1984;52:467-74.

22. Tjan A HL, M iller GD, The J G : Some esthetic factors in a smile. J Prosthet Dent 1984;51:24-28.

23. Lundquist DO, Luther WW. Occlusal plane determination. J Prosthet D ent 1970;23:489-98.

24. Sarmento HR, Dantas RV, Pereira-Cenci T, Faot F. Elements of implant-supported rehabilitation planning in patients with bruxism. J Craniofac Surg. 2012;23(6):1905-09.

\section{ABOUT THE AUTHORS}

\section{Bipin Yeshwantrao Muley (Corresponding Author)}

Postgraduate Student, Department of Prosthodontics, Government Dental College and Hospital, Nagpur, M aharashtra, India, e-mail: bipin1404@gmail.com

\section{Arun N Khalikar}

A ssociate Professor, Department of Prosthodontics, Government Dental College, Nagpur, M aharashtra, India

\section{Sameera Rafique Shaikh}

A ssistant Professor, Department of Prosthodontics, Government Dental College, Nagpur, M aharashtra, India

\section{Mohana M Tagore}

A ssistant Professor, Department of Prosthodontics, Government Dental College, Nagpur, M aharashtra, India

\section{Pravinkumar G Patil}

A ssistant Professor, Department of Prosthodontics, Government Dental College, Nagpur, M aharashtra, India

\section{Sattyam V Wankhade}

A ssistant Professor, Department of Prosthodontics, Government Dental College, Nagpur, M aharashtra, India 